\title{
La actividad física y el rendimiento académico en estudiantes universitarios
}

\section{Physical activity and academic performance in university students}

\author{
Carlos Marcelo Ávila Mediavilla ${ }^{1 *}$, Helder Guillermo Aldas $\operatorname{Arcos}^{1}$ y Santiago Alejandro Jarrín Navas ${ }^{1}$ \\ ${ }^{1}$ Universidad Católica de Cuenca \\ *cjmarce@ hotmail.com
}

DOI: https://doi.org/10.26871/killkana_social.v2i4.214

\begin{abstract}
Resumen
Los profesores de cátedra de las instituciones educativas de educación básica, bachillerato, y superior, en específico en la Educación Superior, han considerado en algunas ocasiones que la Cultura Física, Educación Física, y Actividad Física, no representan un aporte sustancial en la formación integral del estudiante en los niveles de concreción del conocimiento. Pocas son evidencias de los protocolos planteados para atender o entender la relevancia o importancia que puede tener la actividad física en el rendimiento académico de los estudiantes universitarios ecuatorianos. El objetivo fundamental de esta investigación es determinar la relación existente entre la actividad física, para mejorar el rendimiento académico, que constituyen parte fundamental para la formación integral y profesional del estudiante. La investigación es cuantitativa, con alcance exploratorio, socialmente adaptada a involucrar las esferas del conocimiento, psicomotriz, cognitivo, afectivo y social, mediante la aplicación de encuestas a estudiantes y docentes de la UTI, 2014, Quito, se pudo determinar que la actividad física influye significativamente en el rendimiento académico de los estudiantes universitarios.
\end{abstract}

Palabras clave: Actividad física, rendimiento académico, estudiantes universitarios.

\begin{abstract}
The professors of the chairs of the educational institutions of basic, superior and baccalaureate education, even more in Higher Education, have sometimes considered that the physical culture, physical education, and physical activity, do not represent a substantial contribution in the integral formation of the student in the levels of concretion of knowledge. There is no evidence of the protocols proposed to address or understand the relevance or importance that physical activity can have on the academic performance of university students. The fundamental objective of this research is to determine the relationship between physical activity, psychomotor actions that produce caloric expenditure through play, recreational and sports actions to improve physical and mental health and academic performance, which is the ability to respond satisfactorily to educational stimuli developed through the teaching - learning process to obtain a level of functioning and academic achievements over a period of time, which constitute a fundamental part for the integral and professional education of the student. The research is of a contemporary type, socially adapted to involve the spheres of knowledge, psychomotor, cognitive, affective and social, being the case that through surveys to students and teachers, it was determined that physical activity significantly influences academic performance of university students.
\end{abstract}

Key words: Physical activity, academic performance, university students.

\section{Introducción}

En el Ecuador, se han desarrollado varios estudios y planes pilotos para determinar la incidencia de la actividad física en la productividad, innovación, academia y demás aspectos inherentes al ámbito social, enfocado al Buen Vivir de la ciudadanía. El Ministerio de Educación, mediante Acuerdo Ministerial Nro. 020, de 17 de febrero de 2016, incrementó a cinco horas pedagógicas de Educación Física en el nivel de Educación General Básica (Ministerio de Educación, 2016), esto propicio que los estudiantes de estas fajas etareas se activen físicamente en busca de una formación integral. Además se promueve la actividad física, ejercicio o movimiento para que sea la base primordial del desarrollo holístico de los educandos a nivel básico, para promover una política inclusiva e integradora de estrategias pedagógicas y didácticas, que enfatizan la importancia del desarrollo de las capacidades físicas, apropiación de habilidades motrices básicas y mejorar el status psicomotriz, para alcanzar la consolidación de la mente, en el proceso educativo.

A nivel internacional, desde inicios del siglo XXI, ha existido una especial preocupación por las consecuencias derivadas de una limitada práctica deportiva o actividad física, en niños y jóvenes. Los gobernantes de varios paí- 
ses y la Organización Mundial de la Salud (OMS), se encuentran alarmados por la escasa actividad física de la población en general y las consecuencias negativas que ello conlleva como sobrepeso, obesidad, hipertensión arterial, y la diabetes.

(Rejeski y Thompson, 1993), manifiestan:

Estas dos características están mutuamente interrelacionadas, ya que, desde el punto de vista de la salud pública, tan importante es conocer en qué medida la actividad física es beneficiosa para los individuos como saber cuáles son los factores que determinan la existencia de estilos de vida activos, pues si estos no se implantan, los beneficios del ejercicio sobre la salud no llegarán a producirse.

A nivel mundial, el rendimiento académico, en los centros de educación superior, va en decadencia por la acumulación y exageración de estímulos informáticos, propios de la nueva generación como redes sociales, juegos electrónicos, programas de televisión, entre otros, que alejan a los estudiantes de la concentración y atención del aprendizaje, por ello las universidades buscan estrategias para mejorar el desempeño en las aulas.

Mediante estudios realizados en países desarrollados en el ámbito educativo, se puede notar que existe un aumento considerable en recursos económicos y presupuestos destinados a la gestión académica, investigación y vinculación con la sociedad, pero poco ha sido el progreso del rendimiento académico, que obedecen a factores endógenos (atención, percepción, concentración) y exógenos (estímulos tecnológicos, hábitos de vida) de los estudiantes universitarios.

De acuerdo al Ministerio del Deporte del Ecuador en el año 2012, solo el $11 \%$ de la población realiza actividad física, lo que quiere decir que hay un nivel de sedentarismo del 89\%, (Ministerio del Deporte, 2012, p.15). 2003):

De acuerdo a la (Organización Mundial de la Salud,

Se hace referencia al sedentarismo o la falta de actividad física cuando la persona no realiza una cantidad mínima de movimiento diario (por lo menos entre 25 y 30 minutos), que produzca un gasto energético $>10 \%$ del que ocurre habitualmente al llevar a cabo las actividades cotidianas (p. 270).

El sedentarismo es un problema de salud pública, que ha desencadenado en el aumento de enfermedades cardiovasculares en niños, jóvenes, adultos y adultos mayores, que generan al estado una importante inversión en hospitales y agencias gubernamentales para poder restablecer la homeostasis física y mental de los individuos. Es por ello que en las ciudades se ejecutan programas y proyectos de orden social, encaminado a activar a la población, por ejemplo, el ciclo paseo, bici acción, Ecuador ejercítate, Deporte para Todos, entre otros, que permitan ejercitarse por lo menos 3 veces por semana, 30 minutos diarios.

De su parte (Antunes, 2006) afirma:
El ejercicio físico interfiere en el rendimiento cognitivo, debido al aumento de los neuro transmisores y por el cambio de las bases cerebrales. Al lograrse una mejor oxigenación cerebral, el organismo comienza a funcionar mejor, lo que se refleja en el estado de ánimo, la motivación y las ansias de triunfar (párr.5).

Para los autores la actividad física es toda acción psico motriz y socio motriz, que genera gasto calórico, por encima del estado en reposo, puede ser orientada y genera satisfacción, está estrechamente relacionada a la salud física y mental.

Otras fuentes como la (Organización Mundial de la Salud, 2018) se refiere a "La actividad física como cualquier movimiento corporal producido por los músculos esqueléticos que exija gasto de energía.” (párr. 1) y el (Consejo Europeo de Información Nutricional, 2015) lo define "como cualquier movimiento corporal, producido por los músculos esqueléticos, que requiere energía" (párr. 4)

Para (Pizarro, 1985) el rendimiento académico se considera: "una medida de las capacidades que manifiestan en forma positiva lo que una persona ha aprendido como consecuencia de un proceso de formación. El mismo autor, ahora desde una perspectiva propia del alumno, define al rendimiento como una capacidad que responde a los estímulos educativos, susceptibles de ser interpretado según objetivos o propósitos educativos preestablecidos. Este tipo de rendimiento académico puede atenderse en relación a un grupo social que fija los niveles mínimos de aprobación ante un determinado cumulo de conocimientos o aptitudes" (p.31).

En las instituciones educativas de nivel básico, bachillerato y superior del Ecuador se tiene establecida la planificación curricular con carga horaria de por lo menos 5 horas de Educación Física, pero existe poca supervisión por parte del Ministerio de Educación y Ministerio del Deporte, los centros de formación en algunas ocasiones priorizan las asignaturas como la matemática o literatura, dejan relegada y desatendida a la ciencia que permite desarrollar el cuerpo y la mente.

En las universidades, existen pocos datos que permita establecer si la población de estudiantes universitarios realizan actividades físicas orientadas que consten el currículo de las carreras permanentes presenciales, por lo cual este puede ser un factor importante que afecta al rendimiento académico de los estudiantes, ya que la falta de estas actividades pueden desencadenar en sobrepeso, obesidad, hipertensión y diabetes.

Al respecto el autor (Dwyer, 2001), manifiesta: "La relación entre actividad física y desempeño académico; a mayor tiempo de actividad física, mejor rendimiento académico" (párr.4), fundamento al que se adscriben los autores de este estudio.

Al mantener el Concejo de Evaluación, Acreditación y Aseguramiento de la Calidad de la Educación Superior 
(CEAACES), una evaluación permanente y un sistema de clasificación de las universidades en base a criterios de pertinencia, currículo, academia, ambiente institucional y estudiantes, (Consejo de Evaluación, 2015, p.22) propone que la estructura ha iniciado un proceso de fortalecimiento y mejoramiento continuo de los procesos pedagógicos, académicos y administrativos que fomenten un crecimiento sostenido y uniforme en ascenso indudable a la calidad educativa, en los centros de Educación Superior.

El presente estudio permitirá establecer como incide la actividad física en el rendimiento académico de los estudiantes universitarios, diagnosticar el tipo de actividades físicas que realizan los estudiantes universitarios, determinar el nivel de rendimiento académico tienen los estudiantes universitarios, para elaborar una alternativa de propuesta que contribuya a solucionar el problema planteado.

¿Qué tipo de actividades físicas realizan los estudiantes de la universidad?, ¿qué nivel de rendimiento académico tienen los estudiantes de la universidad? ¿existe una alternativa de solución al problema de escases de actividades físicas en el rendimiento académico de los estudiantes universitarios?

Es importante y fundamental presentar estas propuestas investigativas, para entregar a las autoridades de las instituciones una herramienta que permita justificar la creación de dependencias que permitan innovar y crear, en base a evidencias científicas, las propuestas de solución curricular, que cambien el modus vivendi de los estudiantes dentro y fuera de las facultades, para motivar al desarrollo integral de los estudiantes alcanzan niveles óptimos de atención, concentración y pro actividad, desencadenándose en la excelencia académica, para entregar profesionales altamente eficientes con una visión humana e integradora.

Los beneficiarios de esta propuesta, son alrededor de medio millón de estudiantes que se encuentran matriculados en el sistema universitario, además se tendrá que considerar los futuros jóvenes que formen parte de la prestigiosa institución, puesto que se podrá atender a sus necesidades reales de actividades físicas, orientadas a mejorar su calidad de vida y su rendimiento académico.

La utilidad teórica - práctica, está fundamentada básicamente en obtener las necesidades y expectativas de los estudiantes universitarios, que forman parte del pensum académico actual, y en base a su experiencia buscan tener alguna alternativa diferente para ocupar su tiempo libre o que a su vez, soliciten que la actividad física orientada por un profesional, sea parte activa de su formación, se considera que los resultados y estadísticas son contundentes, se tendrá la posibilidad de proponer a las autoridades pertinentes la creación de una dependencia que brinde la oportunidad.

Los profesionales en Ciencias de la Actividad Física, Deportes y Recreación, consideran que el interés se fundamenta en fomentar y fortalecer la formación de los estudiantes, optimizan el desarrollo integral, articulan el aspecto psicomotor y cognoscitivo y, para implementar pro- gramas de actividad física orientados, ofrecen oportunidad laboral a profesionales, monitores y entrenadores en los nuevos programas deportivos.

Las universidades, centros, que brindan el servicio académico, tiene claramente definida su misión, que es formar seres humanos integrales con capacidad emprendedora y de liderazgo, mediante una educación de calidad, impulsan la investigación para contribuir al desarrollo de la sociedad y la visión que pretende ser líder y referente positivo de gestión educativa de calidad por su contribución efectiva a la sociedad, por lo tanto esta propuesta promueve la consecución para que se cumplan los objetivos planteados.

\section{Metodología}

Para la presente investigación se ha considerado el enfoque cuantitativo ya que nos permitirá cuantificar las variables a ser investigadas, es netamente de impacto social, comportamental y procedimental de estudiantes.

En el tema de investigación la actividad física y el rendimiento académico, es una investigación de carácter social, por tratarse de problemas institucionales, su evolución beneficiará a todos los estudiantes universitarios del país.

La población, se determina en la totalidad de sujetos a ser investigados, deben tener condiciones o características similares que nos ayuden a que los datos e información alcanzada sea la más confiable.

Los sujetos a ser investigados fueron estudiantes universitarios, considerados de ambos géneros, de entre 18 y 35 años de edad en su mayoría, y que se encuentran en el programa de pregrado en modalidad presencial.

Para la presente investigación se aplicó un cuestionario, para evaluar la Variable Independiente: Actividad Física y la Variable Dependiente: Rendimiento Académico, tanto a los estudiantes y docentes universitarios.

El procesamiento de la información se realizó en base a una revisión crítica de resultados, codificación de resultados, tabulación de resultados, se explica mediante cuadros de frecuencias y porcentajes los resultados obtenidos de la investigación para posteriormente realizar gráficos estadísticos que coadyuven a la presentación clara y objetiva de los resultados.

\section{Resultados}

Los actores principales del proceso enseñanza - aprendizaje en la educación superior son los estudiantes y docentes, por ello, se aplicó una encuesta, con reactivos que integran las variables de investigación, a continuación, el detalle de los resultados evidenciados, luego de aplicar el instrumento a los estudiantes universitarios de la UTI 2014 Quito:

1. ¿Cree usted, que las actividades físicas mejoran su rendimiento académico? 


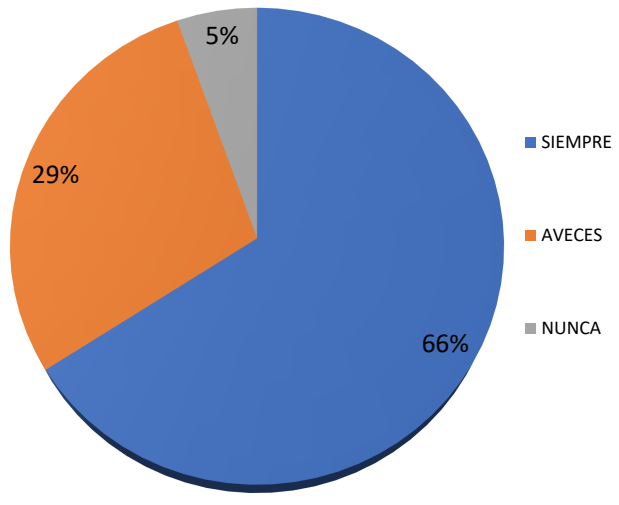

Figura 1. Actividades físicas mejoran su rendimiento académico Elaborado por: Marcelo Ávila

Los estudiantes universitarios, consideran que actividades físicas mejoran su rendimiento académico, el resultado el siguiente: siempre $66 \%$, a veces $29 \%$ y nunca $5 \%$.

Un alto porcentaje de estudiantes considera que la actividad física, es un factor preponderante en mejorar el rendimiento académico, se considera que tiene múltiples beneficios, y en la universidad no tienen esta oferta.

2. ¿Considera usted, que se debe implementar horas pedagógicas de Educación Física o Clubes deportivos, en el pensum académico de las Facultades?

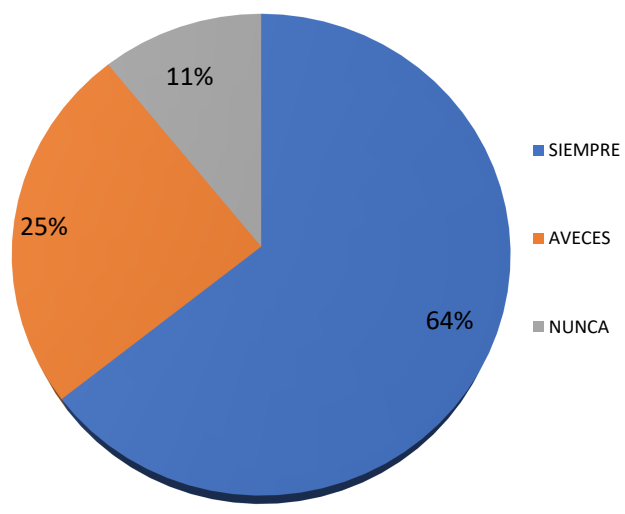

Figura 2. Implementar horas pedagógicas de Educación Física o Clubes deportivos, en el pensum académico de las Facultades

Elaborado por: Marcelo Ávila

Los estudiantes universitarios, consideran que implementar horas pedagógicas de Educación Física o Clubes deportivos, en el pensum académico de las Facultades, el resultado el siguiente: siempre $64 \%$, a veces $25 \%$ y nunca $11 \%$.

A gran escala se puede evidenciar, la tendencia de los estudiantes, que tienen la necesidad de implementar las horas pedagógicas de Educación Física, se considera, que este factor puede ser determinante, para la motivación e impulso para cumplir los objetivos planteados como estudiantes.

Así mismo se realizó la encuesta a los docentes universitarios, se evidenció lo siguiente:
1. ¿Cree usted, que las actividades físicas mejoran el rendimiento académico?

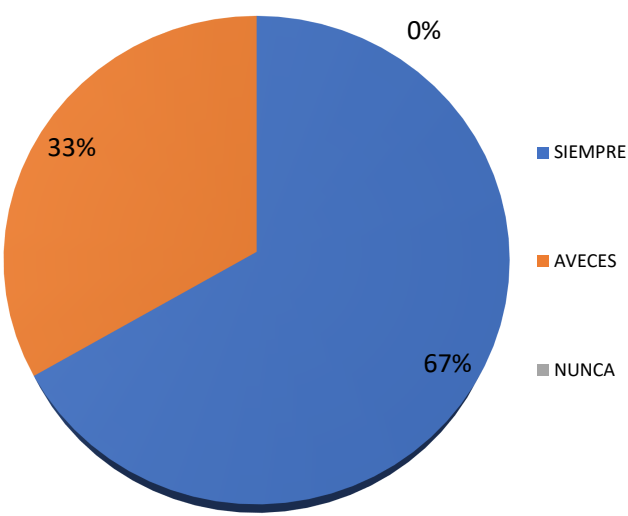

Figura 3. Actividades físicas mejoran su rendimiento académico

Elaborado por: Marcelo Ávila

Los docentes universitarios, consideran que actividades físicas mejoran el rendimiento académico de los estudiantes, el resultado el siguiente: siempre $67 \%$, a veces $33 \%$ y nunca $0 \%$.

Un alto porcentaje de estudiantes considera que la actividad física, es un factor preponderante en mejorar el rendimiento académico, consideran que tiene múltiples beneficios.

2. ¿Considera usted, que se debe implementar horas pedagógicas de Educación Física o Clubes deportivos, en el pensum académico de las Facultades?

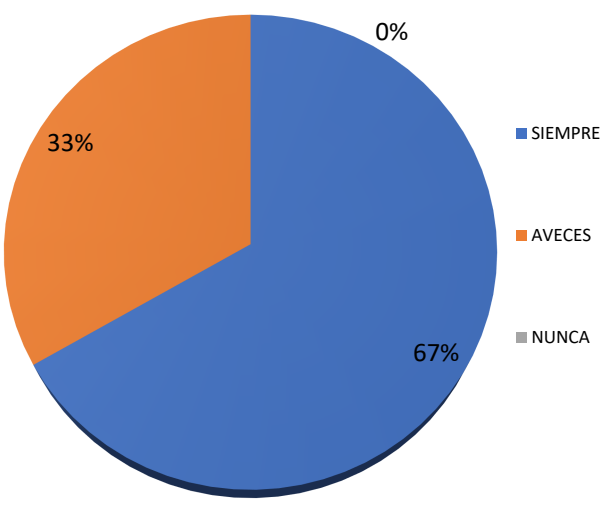

Figura 4. Implementar horas pedagógicas de Educación Física Elaborado por: Marcelo Ávila

Los docentes universitarios, consideran que implementar horas pedagógicas de Educación Física o Clubes deportivos, en el pensum académico de las Facultades, el resultado el siguiente: siempre 67

Los docentes manifiestan que sería una gran oportunidad para los estudiantes, de tener la carga horaria programada de Educación Física y Deportes, en la malla curricular, pues manifiestan que sería un factor importante para la formación holística de los estudiantes. 


\section{Conclusiones}

El tipo de actividades físicas que realizan los estudiantes está supeditado, al altísimo grado de sedentarismo, es decir, que no se realiza ejercicios específicos o movimientos musculo esqueléticos, planificados u orientados, no existe periodicidad de estas actividades y si se realiza algún tipo de actividad física, son básicamente para labores cotidianas, laborales y escolares, en la Universidad, no hay la posibilidad de realizar actividad física, periódicamente, en vista, que no existe una asignatura de educación física o clubes deportivos, en el currículo académico, que brinde la oportunidad y la oferta a los estudiantes.

El nivel de rendimiento académico de los estudiantes que cursan asignaturas en la universidad, es aceptable, ya que la formación académica y andragógica es prioridad, en esta institución educativa, pues luego de la evaluación a la cual fue sometida por las instituciones gubernamentales pertinentes, se ubicó en la categoría B, una calificación muy buena a nivel nacional.

Existe algunas alternativas de solución, para la escases de actividades físicas en el rendimiento académico, de los estudiantes, las más relevantes, es permitir a los estudiantes, tener un régimen de actividad física mediante, la implementación de horas curriculares de educación física, que permitirá, mejorar el estatus psicomotriz de los jóvenes, además de ofrecer clubes deportivos con diferentes disciplinas deportivas, para aplicar la metodología del entrenamiento deportivo y las estrategias metodológicas, para desarrollar las capacidades físicas y coordinativas.

\section{Recomendaciones}

Los estudiantes deben tener la oportunidad y oferta deportiva, de horas curriculares de educación física, acondicionamiento físico, clases de deportes específicos en el proceso de enseñanza - aprendizaje, en los cuales, tengan un enfoque recreativo y deportivo, que permita a los estudiantes, tener alternativas, de movimiento musculo esquelética y cardiovascular. Se considera que la formación integral de los estudiantes, les permitirá ser críticos, innovadores y productivos.

$\mathrm{Si}$ bien es cierto que el rendimiento académico, de los estudiantes es bueno, pero deben apuntalar algunos procesos, estrategias y procedimientos, para poder articular el trabajo físico, con el trabajo académico. El priorizar algunas actividades físicas y el incremento de un pensum académico en las horas de educación física, debido a que los estudiantes buscan potencializar estratégicamente sus habilidades y destrezas, buscan la formación integral del ser humano.

La propuesta alternativa de solución, para la escases de actividades físicas en el rendimiento académico, de los estudiantes universitarios, es permitir a los estudiantes, tener un régimen de actividad física mediante, la implementación de horas andragógicas de educación física, que permitirá, mejorar el estatus psicomotriz de los jóvenes, además de ofrecer clubes deportivos con diferentes disciplinas deportivas, para crear un departamento de educación física, con una estructura de profesionales en el área, que planifiquen y ejecuten las programas, para aplicar la metodología del entrenamiento deportivo y las estrategias metodológicas, para desarrollar las capacidades físicas y coordinativas, que coadyuva a alcanzar el perfil profesional, encaminado a ser altamente comprometido con la patria, la institución y la familia.

\section{Referencias Bibliográficas}

Antunes, S. (2006). Reviewing on physical exercise and the cognitive function. (Revisión sobre ejercicio físico y la función cognitiva) (Vol. 12).

Consejo de Evaluación, A. y. A. d. 1. C. d. 1. E. (2015). Modelo Genérico de Evaluación del Entorno de Aprendizaje de Carreras en Ecuador.

Consejo Europeo de Información Nutricional. (2015). Actividad Física y Salud.

Dwyer, T. (2001). Relation of academic perfomance to physical activity and fitnes in children. (Relación del rendimiento académico y la actividad física y fitness en niños).

Ministerio de Educación. (2016). Acuerdo Ministerial Nro. 020 .

Ministerio del Deporte. (2012). El deporte en cifras. Ecuador.

Organización Mundial de la Salud. (2003). Estrategia mundial sobre régimen alimentario, actividad física y salud.

Organización Mundial de la Salud. (2018). Estrategia mundial sobre régimen alimentario, actividad física y salud.

Pizarro, R. (1985). Rasgos y actitudes del profesor efectivo.

Rejeski, W., y Thompson. (1993). A historical and conceptual roots of exercise psychology. (Historia $y$ conceptos de la psicología del ejercicio). Nueva York.

Recibido: 22 de febrero de 2018

Aceptado: 6 de diciembre de 2018 
